\title{
Tryon's Conjecture and Energy and Momentum of Bianchi Type Universes
}

\author{
Prajyot Kumar Mishra, ${ }^{1}$ Bibhudatta Panda, ${ }^{1}$ Pradosh Ranjan Pattanayak, ${ }^{2}$ \\ and Sunil Kumar Tripathy ${ }^{3}$ \\ ${ }^{1}$ Department of Electrical Engineering, Indira Gandhi Institute of Technology, Sarang, Dhenkanal District, Odisha 759146, India \\ ${ }^{2}$ Department of Computer Science Engineering and Applications, Indira Gandhi Institute of Technology, Sarang, \\ Dhenkanal District, Odisha 759146, India \\ ${ }^{3}$ Department of Physics, Indira Gandhi Institute of Technology, Sarang, Dhenkanal District, Odisha 759146, India
}

Correspondence should be addressed to Sunil Kumar Tripathy; tripathy_sunil@rediffmail.com

Received 5 February 2016; Revised 16 April 2016; Accepted 5 May 2016

Academic Editor: Edward Sarkisyan-Grinbaum

\begin{abstract}
Copyright (C) 2016 Prajyot Kumar Mishra et al. This is an open access article distributed under the Creative Commons Attribution License, which permits unrestricted use, distribution, and reproduction in any medium, provided the original work is properly cited. The publication of this article was funded by $\mathrm{SCOAP}^{3}$.
\end{abstract}

The energy and momentum of some diagonal anisotropic Bianchi type Universes are obtained using different energy-momentum complexes in the framework of General Relativity. The Møller energy is found to be zero for all the Universes considered in the present work. In all other prescriptions, the energy and momentum vanish when the sum of the metric parameters $\alpha$ and $\beta$ vanishes. This result obviously raises a question: why this specific choice? We have explored Tryon's conjecture that the Universe must have a zero net value for all conserved quantities to get some ideas on this issue.

\section{Introduction}

The General Relativity (GR) as formulated by Einstein is now a hundred years old, but the problem of energymomentum localization in GR has not yet been settled. Einstein conceived the idea of covariant conservation of energy and momenta of gravitational fields along with those of matter and nongravitational fields [1]. However, quantities like energy and momentum at any local point of a manifold should always be conserved as per the usual conservation law $T_{i, j}^{j}=0$, where $T_{i}^{j}$ is the energy-momentum tensor and the comma (,) denotes an ordinary differentiation. The covariant formulation requires nontensorial fields. Obviously, the energy-momentum tensor due to the gravitational field turns out to be nontensorial (pseudotensor). The choice of this pseudotensor is not unique and therefore it has led to the formulation of a number of prescriptions for the calculation of energy and momentum [2-9]. The interesting thing about these prescriptions is that they depend on the coordinate systems used. It has been observed earlier that, for
quasi-Cartesian coordinates, all the prescriptions can provide some reasonable and meaningful results. However, some coordinates independent energy-momentum complexes have been proposed by Møller [7], Komar [8], and Penrose [9]. But some of these coordinates independent prescriptions are questioned for their limited applicability.

The issue of energy localization has been widely discussed in the literature in the framework of both GR and teleparallel gravity. Misner et al. showed that energy is localized only for spherically symmetric systems [10]. Cooperstock and Sarracino counter commented on the idea of Misner and established that if energy is localized in spherically symmetric systems then it can be localized in any space-time [11]. Bondi perceived that a nonlocalizable form of energy is not admissible in GR, because any form of energy contributes to gravitation and therefore its location can in principle be found [12]. Virbhadra and his collaborators revived the debate and proved that energy-momentum complexes coincide and give reasonable results for some well known and physically significant space-times [13-21]. Virbhadra showed 
that different prescriptions can provide the same result for Kerr-Schild space-time when Cartesian coordinates are used [13]. Following Virbhadra, many researchers obtained interesting results on this pressing issue of energy localization [2239]. Contrary to the previous results, Gad explored the failure of these prescriptions to provide similar results in some specific examples of space-time [40-43]. The issue of energy localization has also been considered in the framework of teleparallel gravity [44-49]. It has been concluded in some recent works that the energy-momentum definitions are identical not only in GR but also in teleparallel gravity [48, $50,51]$.

Tryon anticipated the net energy of the Universe to be zero [52]. Albrow [53] also had a similar assumption on the net energy of the Universe. From the calculation of energy of a closed homogeneous isotropic Friedmann-RobertsonWalker (FRW) Universe, Rosen showed that the total energy of the Universe is zero everywhere [54]. Cooperstock and Israelit [55] and Johri et al. [56] also found similar results for closed FRW Universe. Vargas calculated the energy and momentum of FRW Universe in Landau-Lifshitz and Einstein prescriptions in the context of teleparallel gravity and obtained the total energy of the Universe to be zero. In a recent work, Tripathy et al. [57] have obtained the energy and momentum of Bianchi type $\mathrm{VI}_{h}\left(\mathrm{BVI}_{h}\right)$ Universes in the framework of GR in different prescriptions and have shown that the results can agree only for a specific value of the metric parameter $h$. They have also raised the question, on the basis of Tryon's conjecture, of why the $\mathrm{BVI}_{h}$ spacetime requires the specific value of the parameter. In the present work, we have tried to investigate the question raised by Tripathy et al. by considering anisotropic Bianchi type Universes. It is worth mentioning here that anisotropic spacetimes are more interesting to investigate in the context of recent observations. Many authors have taken interest in the calculation of the energy and momentum of anisotropic Universe in recent times. Using different energy-momentum complexes either in GR or in teleparallel gravity, Banerjee and Sen [58], Xulu [59], and Aydogdu and Salti [46] have obtained the total energy of Bianchi type I (BI) Universes to be zero everywhere. Aydogdu and Salti have also calculated the energy of LRS Bianchi II Universe to get consistent results [60]. Radinschi calculated the energy distribution of a Bianchi type $\mathrm{VI}_{0}\left(\mathrm{BVI}_{0}\right)$ Universe using Tolman, BergmannThompson, and Møller prescriptions and found the total energy of the Universe to be zero [61]. In another work, Radinschi calculated the energy of $\mathrm{BVI}_{0}$ Universe using the Landau-Lifshitz, Papapetrou, and Weinberg prescriptions and found similar results [62]. Aygün and Tarhan have obtained the energy and momentum of Bianchi IV Universe in different energy-momentum complexes in the framework of both GR and teleparallel gravity [63].

The organisation of the paper is as follows. In Section 2, we present the basics of diagonal anisotropic Bianchi type (DB) Universes. In Section 3, the energy and momentum densities for these Universes are obtained using some well known prescriptions. Results of the present work are discussed and analysed based upon Tryon's conjecture advocating a null total energy state of the Universe in Section 4. The summary and conclusion are presented at the end, in Section 5. In the present work, we have used the convention that the Latin indices take values from 0 to 3 and Greek indices run from 1 to 3 . Also, we have used the geometrized unit system where $8 \pi G=c=1$, with $G$ and $c$ being the Newtonian gravitational constant and speed of light in vacuum, respectively.

\section{Diagonal Anisotropic Bianchi Type Universe}

The Universe is observed to be mostly isotropic and can be well explained by $\Lambda$ CDM ( $\Lambda$ dominated Cold Dark Matter) model. However, certain measurements of cosmic microwave background from Wilkinson Microwave Anisotropic Probe (WMAP) show some anomalous features of $\Lambda$ CDM model at large scale [64]. These precise measurements suggest an asymmetric expansion of the Universe with one direction expanding in different manner than the other two transverse directions [65-67]. The Planck data [68-71] shows slight red shifting of the primordial power spectrum of curvature perturbation from exact scale invariance. It can be inferred from the Planck data that $\Lambda$ CDM model cannot be a good fit at least at high multipoles. The issue of global anisotropy can be dealt with in many ways. However, a simple way is to modify the FRW model by considering asymmetric expansion along different spatial directions. In this sense, Bianchi type models play important roles. The Bianchi type models are homogeneous space-times having anisotropic spatial sections and are exact solutions of Einstein field equations. In the present work, we have considered diagonal anisotropic Bianchi type Universes modelled through the metric

$$
\begin{aligned}
d s^{2}= & -d t^{2}+A^{2}(t) d x^{2}+B^{2}(t) e^{2 \alpha x} d y^{2} \\
& +C^{2}(t) e^{2 \beta x} d z^{2},
\end{aligned}
$$

where $A, B$, and $C$ are the directional scale factors and are considered as functions of cosmic time $t$ only. The exponents $\alpha$ and $\beta$ are constants of time and can assume any real values compatible with the physical Universe. It is worth mentioning here that the models are not vacuum solutions and can have nonvanishing components of the energy-momentum tensor $T_{k}^{i} \neq 0$.

The determinant of the metric tensor for the DB spacetime in (1) is $g=\left|g_{a b}\right|=-A^{2} B^{2} C^{2} e^{2(\alpha+\beta) x}$. The nonvanishing covariant components of the metric tensor are $g_{00}=-1$, $g_{11}=A^{2}, g_{22}=B^{2} e^{2 \alpha x}$, and $g_{33}=C^{2} e^{2 \beta x}$. The corresponding contravariant components are

$$
\begin{aligned}
& g^{00}=-1, \\
& g^{11}=\frac{1}{A^{2}}, \\
& g^{22}=\frac{1}{B^{2} e^{2 \alpha x}}, \\
& g^{33}=\frac{1}{C^{2} e^{2 \beta x}} .
\end{aligned}
$$


TABLE 1: Different energy-momentum prescriptions used in the present work.

\begin{tabular}{|c|c|c|c|}
\hline Prescription & Energy-momentum pseudotensor & Densities & Energy-momentum four vectors $\left(P_{i}\right)$ \\
\hline Einstein & $\begin{array}{l}\Theta_{i}^{k}=\frac{1}{16 \pi} H_{i, l}^{k l} \\
H_{i}^{k l}=-H_{i}^{l k}=\frac{g_{i n}}{\sqrt{-g}}\left[-g\left(g^{k n} g^{l m}-g^{l n} g^{k m}\right)\right]_{, m}\end{array}$ & $\Theta_{0}^{0}, \Theta_{\alpha}^{0}$ & $\Theta_{i}^{0} d x^{1} d x^{2} d x^{3}$ \\
\hline Landau-Lifshitz & $\begin{array}{l}L^{i k}=\frac{1}{16 \pi} \lambda_{, l m}^{i k l m} \\
\lambda^{i k l m}=-g\left(g^{i k} g^{l m}-g^{i l} g^{k m}\right)\end{array}$ & $L^{00}, L^{\alpha 0}$ & $L^{i 0} d x^{1} d x^{2} d x^{3}$ \\
\hline Papapetrou & $\begin{array}{l}\Sigma^{i k}=\frac{1}{16 \pi} \mathcal{N}_{, l m}^{i k l m} \\
\mathscr{N}^{i k l m}=\sqrt{-g} \times\left(g^{i k} \eta^{l m}-g^{i l} \eta^{k m}+g^{l m} \eta^{i k}-g^{l k} \eta^{i m}\right)\end{array}$ & $\Sigma^{00}, \Sigma^{\alpha 0}$ & $\Sigma^{i 0} d x^{1} d x^{2} d x^{3}$ \\
\hline Bergmann-Thompson & $\begin{array}{l}\mathbf{B}^{i k}=\frac{1}{16 \pi}\left[g^{i l} \mathscr{B}_{l}^{k m}\right]_{, m} \\
\mathscr{B}_{l}^{k m}=\frac{g_{l n}}{\sqrt{-g}}\left[-g\left(g^{k n} g^{m p}-g^{m n} g^{k p}\right)\right]_{, p}\end{array}$ & $\mathbf{B}^{00}, \mathbf{B}^{\alpha 0}$ & $\mathbf{B}^{i 0} d x^{1} d x^{2} d x^{3}$ \\
\hline Møller & $\begin{aligned} T_{i}^{k} & =\frac{1}{8 \pi} \chi_{i, l}^{k l} \\
\chi_{i}^{k l} & =-\chi_{i}^{l k}=\sqrt{-g}\left[g_{i n, m}-g_{i m, n}\right] g^{k m} g^{n l}\end{aligned}$ & $T_{0}^{0}, T_{\alpha}^{0}$ & $T_{i}^{0} d x^{1} d x^{2} d x^{3}$ \\
\hline
\end{tabular}

\section{Energy-Momentum Complexes}

We have calculated the energy and momentum of DB Universes described by metric (1) using the Einstein, LandauLifshitz, Papapetrou, Bergmann-Thompson, and Møller prescriptions. The definitions of the energy-momentum pseudotensors and corresponding energy-momentum four vectors $\left(P_{i}\right)$ for different prescriptions are given in Table 1. We consider the definitions of the well known energymomentum prescriptions in the framework of General Relativity. In the following subsections, we report the nonvanishing components of the super potentials and the consequent energy and momentum densities. The calculated energy and momentum densities for the DB Universes are given in Table 2. The results for energy and momentum densities are presented in general forms of the directional scale factors $A$, $B$, and $C$ and the exponents $\alpha$ and $\beta$. From these general results, the energy and momentum of a given diagonal Bianchi type Universe can be obtained in straightforward manner by incorporating the time dependence of the scale factors and the values of the exponents $\alpha$ and $\beta$.

3.1. Einstein Energy-Momentum Complex. The required nonvanishing components of $H_{i}^{k l}$ are

$$
\begin{aligned}
& H_{0}^{01}=-H_{0}^{10}=2(\alpha+\beta) \frac{B C}{A} e^{(\alpha+\beta) x}, \\
& H_{1}^{01}=-H_{1}^{10}=2 A B C\left(\frac{\dot{B}}{B}+\frac{\dot{C}}{C}\right) e^{(\alpha+\beta) x}, \\
& H_{2}^{02}=-H_{2}^{20}=2 A B C\left(\frac{\dot{A}}{A}+\frac{\dot{C}}{C}\right) e^{(\alpha+\beta) x}, \\
& H_{2}^{21}=-H_{2}^{12}=\frac{2 B C \beta}{A} e^{(\alpha+\beta) x}, \\
& H_{3}^{03}=-H_{3}^{30}=2 A B C\left(\frac{\dot{A}}{A}+\frac{\dot{B}}{B}\right) e^{(\alpha+\beta) x}, \\
& H_{3}^{31}=-H_{3}^{13}=\frac{2 B C \alpha}{A} e^{(\alpha+\beta) x} .
\end{aligned}
$$

Here, a dot over a field variable denotes differentiation with respect to time.

The components of energy and momentum densities can now be obtained as

$$
\begin{aligned}
& \Theta_{0}^{0}=\frac{(\alpha+\beta)^{2}}{8 \pi} \frac{B C}{A} e^{(\alpha+\beta) x}, \\
& \Theta_{1}^{0}=\frac{(\alpha+\beta)}{8 \pi} A B C\left(\frac{\dot{B}}{B}+\frac{\dot{C}}{C}\right) e^{(\alpha+\beta) x}, \\
& \Theta_{2}^{0}=\Theta_{3}^{0}=0 .
\end{aligned}
$$

3.2. Landau and Lifshitz Energy-Momentum Complex. The nonvanishing components of $\lambda^{i k l m}$ are obtained as

$$
\begin{aligned}
& \lambda^{0011}=\lambda^{1100}=-\lambda^{1010}=-\lambda^{0101}=-B^{2} C^{2} e^{2(\alpha+\beta) x}, \\
& \lambda^{0022}=\lambda^{2200}=-\lambda^{2020}=-\lambda^{0202}=-A^{2} C^{2} e^{2 \beta x}, \\
& \lambda^{1122}=\lambda^{2211}=-\lambda^{1212}=-\lambda^{2121}=C^{2} e^{2 \beta x}, \\
& \lambda^{2233}=\lambda^{3322}=-\lambda^{2323}=-\lambda^{3232}=A^{2}, \\
& \lambda^{1133}=\lambda^{3311}=-\lambda^{1313}=-\lambda^{3131}=B^{2} e^{2(\alpha+\beta) x}, \\
& \lambda^{0033}=\lambda^{3300}=-\lambda^{3030}=-\lambda^{0303}=-A^{2} B^{2} e^{2 \alpha x} .
\end{aligned}
$$

Consequently, the energy and momentum densities in the Landau and Lifshitz prescription become

$$
\begin{aligned}
& L^{00}=-\frac{(\alpha+\beta)^{2}}{4 \pi} B^{2} C^{2} e^{2(\alpha+\beta) x}, \\
& L^{10}=\frac{(\alpha+\beta)}{4 \pi} B^{2} C^{2}\left(\frac{\dot{B}}{B}+\frac{\dot{C}}{C}\right) e^{2(\alpha+\beta) x}, \\
& L^{20}=L^{30}=0 .
\end{aligned}
$$


TABLE 2: Energy and momentum densities of diagonal Bianchi type Universes using different energy-momentum prescriptions used in the present work.

\begin{tabular}{|c|c|c|}
\hline Prescription & Energy density & Momentum densities \\
\hline Einstein & $\Theta_{0}^{0}=\frac{(\alpha+\beta)^{2}}{8 \pi} \frac{B C}{A} e^{(\alpha+\beta) x}$ & $\begin{array}{l}\Theta_{1}^{0}=\frac{(\alpha+\beta)}{8 \pi} A B C\left(\frac{\dot{B}}{B}+\frac{\dot{C}}{C}\right) e^{(\alpha+\beta) x}, \\
\Theta_{2}^{0}=\Theta_{3}^{0}=0 .\end{array}$ \\
\hline Landau-Lifshitz & $L^{00}=-\frac{(\alpha+\beta)^{2}}{4 \pi} B^{2} C^{2} e^{2(\alpha+\beta) x}$ & $\begin{array}{l}L^{10}=\frac{(\alpha+\beta)}{4 \pi} B C(\dot{B} C+B \dot{C}) e^{2(\alpha+\beta) x} \\
L^{20}=L^{30}=0 .\end{array}$ \\
\hline Papapetrou & $\Sigma^{00}=-\frac{(\alpha+\beta)^{2}}{16 \pi}\left(1+\frac{1}{A^{2}}\right) A B C e^{(\alpha+\beta) x}$ & $\begin{aligned} \Sigma^{10}= & \frac{(\alpha+\beta)}{16 \pi}\left(\frac{\dot{A}}{A}+\frac{\dot{B}}{B}+\frac{\dot{C}}{C}\right) A B C e^{(\alpha+\beta) x} \\
& +\frac{(\alpha+\beta)}{16 \pi}\left(\frac{1}{A^{2}}\right)\left(\frac{\dot{B}}{B}+\frac{\dot{C}}{C}-\frac{\dot{A}}{A}\right) A B C e^{(\alpha+\beta) x}, \\
\Sigma^{20}= & \Sigma^{30}=0 .\end{aligned}$ \\
\hline Bergmann-Thompson & $\mathbf{B}^{00}=-\frac{(\alpha+\beta)^{2}}{8 \pi} \frac{B C}{A} e^{(\alpha+\beta) x}$ & $\begin{array}{l}\mathbf{B}^{10}=\frac{(\alpha+\beta)}{8 \pi} \frac{B C}{A}\left(\frac{\dot{B}}{B}+\frac{\dot{C}}{C}\right) e^{(\alpha+\beta) x} \\
\mathbf{B}^{20}=\mathbf{B}^{30}=0\end{array}$ \\
\hline Møller & $T_{0}^{0}=0$ & $\begin{array}{l}T_{1}^{0}=-\frac{(\alpha+\beta)}{4 \pi} \dot{A} B C e^{(\alpha+\beta) x} \\
T_{2}^{0}=T_{3}^{0}=0\end{array}$ \\
\hline
\end{tabular}

3.3. Papapetrou Energy-Momentum Complex. The required nonvanishing components of $\mathscr{N}^{i k l m}$ are

$$
\begin{aligned}
& \mathscr{N}^{1001}=\mathscr{N}^{2002}=\mathcal{N}^{3003}=A B C e^{(\alpha+\beta) x}, \\
& \mathcal{N}^{0011}=\mathcal{N}^{1100}=-\left(1+\frac{1}{A^{2}}\right) A B C e^{(\alpha+\beta) x}, \\
& \mathscr{N}^{0110}=\mathcal{N}^{1010}=\left(\frac{B C}{A}\right) e^{(\alpha+\beta) x}, \\
& \mathcal{N}^{0022}=\mathcal{N}^{2200}=-\left(1+\frac{1}{B^{2} e^{2 \alpha x}}\right) A B C e^{(\alpha+\beta) x}, \\
& \mathcal{N}^{0330}=\mathscr{N}^{3030}=\frac{A B}{C} e^{(\alpha-\beta) x}, \\
& \mathscr{N}^{0220}=\mathcal{N}^{2020}=\left(\frac{A C}{B}\right) e^{(\beta-\alpha) x}, \\
& \mathcal{N}^{1122}=\mathcal{N}^{2211}=\left(\frac{1}{B^{2} e^{2 \alpha x}}+\frac{1}{A^{2}}\right) A B C e^{(\alpha+\beta) x}, \\
& \mathcal{N}^{1221}=\mathcal{N}^{2121}=-\left(\frac{A C}{B}\right) e^{(\beta-\alpha) x}, \\
& \mathcal{N}^{1331}=\mathcal{N}^{3131}=-\left(\frac{A B}{C}\right) e^{(\alpha-\beta) x}, \\
& \mathcal{N}^{1313}=\mathcal{N}^{3113}=-\left(\frac{B C}{A}\right) e^{(\alpha+\beta) x}, \\
& \mathscr{N}^{1133}=\mathcal{N}^{3311}=\left(\frac{1}{A^{2}}+\frac{1}{C^{2} e^{2 \beta x}}\right) A B C e^{(\alpha+\beta) x}, \\
& \mathcal{N}^{2112}=-\mathcal{N}^{1212}=-\left(\frac{B C}{A}\right) e^{(\alpha+\beta) x}, \\
& \mathcal{N}^{2332}=\mathcal{N}^{3232}=-\left(\frac{A B}{C}\right) e^{(\alpha-\beta) x}, \\
& \mathcal{N}^{3300}=\mathscr{N}^{0033}=-\left(1+\frac{1}{C^{2} e^{2 \beta x}}\right) A B C e^{(\alpha+\beta) x}, \\
& \mathscr{N}^{3322}=\mathscr{N}^{2233}=\left(\frac{1}{C^{2} e^{2 \beta x}}+\frac{1}{B^{2} e^{2 \alpha x}}\right) A B C e^{(\alpha+\beta) x} .
\end{aligned}
$$

The energy density and momentum density components in the Papapetrou prescription are obtained from (7) as

$$
\begin{aligned}
\Sigma^{00} & =-\frac{(\alpha+\beta)^{2}}{16 \pi}\left(1+\frac{1}{A^{2}}\right) A B C e^{(\alpha+\beta) x}, \\
\Sigma^{10} & =\frac{(\alpha+\beta)}{16 \pi}\left[\left(\frac{\dot{A}}{A}+\frac{\dot{B}}{B}+\frac{\dot{C}}{C}\right)\right. \\
& \left.+\left(\frac{1}{A^{2}}\right)\left(\frac{\dot{B}}{B}+\frac{\dot{C}}{C}-\frac{\dot{A}}{A}\right)\right] A B C e^{(\alpha+\beta) x}, \\
\Sigma^{20} & =0 \\
\Sigma^{30} & =0 .
\end{aligned}
$$

3.4. Bergmann-Thompson Energy-Momentum Complex. The nonvanishing components of $\mathscr{B}_{l}^{k m}$ are

$$
\begin{aligned}
& \mathscr{B}_{0}^{01}=-\mathscr{B}_{0}^{10}=\mathscr{B}_{3}^{31}=-\mathscr{B}_{3}^{13}=\frac{2 B C \alpha}{A} e^{(\alpha+\beta) x}, \\
& \mathscr{B}_{1}^{01}=-\mathscr{B}_{1}^{10}=2 A(\dot{B} C+B \dot{C}) e^{(\alpha+\beta) x}, \\
& \mathscr{B}_{2}^{02}=-\mathscr{B}_{2}^{20}=2 B(\dot{A} C+A \dot{C}) e^{(\alpha+\beta) x}, \\
& \mathscr{B}_{3}^{03}=-\mathscr{B}_{3}^{30}=2 C(\dot{A} B+A \dot{B}) e^{(\alpha+\beta) x} .
\end{aligned}
$$

Using (9), the energy and momentum density components, that is, $\mathbf{B}^{00}$ and $\mathbf{B}^{\alpha 0}$, can be obtained as

$$
\begin{aligned}
& \mathbf{B}^{00}=-\frac{(\alpha+\beta)^{2}}{8 \pi} \frac{B C}{A} e^{(\alpha+\beta) x}, \\
& \mathbf{B}^{10}=\frac{(\alpha+\beta)}{8 \pi} \frac{B C}{A}\left(\frac{\dot{B}}{B}+\frac{\dot{C}}{C}\right) e^{(\alpha+\beta) x} \\
& \mathbf{B}^{20}=\mathbf{B}^{30}=0 .
\end{aligned}
$$


3.5. Møller Energy-Momentum Complex. The nonvanishing components of $\chi_{i}^{k l}$ are

$$
\begin{aligned}
& \chi_{1}^{01}=-\chi_{1}^{10}=-2 \dot{A} B C e^{(\alpha+\beta) x}, \\
& \chi_{2}^{02}=-\chi_{2}^{20}=-2 A \dot{B} C e^{(\alpha+\beta) x}, \\
& \chi_{3}^{03}=-\chi_{3}^{30}=-2 A B \dot{C} e^{(\alpha+\beta) x}, \\
& \chi_{2}^{21}=-\chi_{2}^{12}=-\frac{2 B C \alpha}{A} e^{(\alpha+\beta) x}, \\
& \chi_{3}^{31}=-\chi_{3}^{13}=-\frac{2 B C \beta}{A} e^{(\alpha+\beta) x} .
\end{aligned}
$$

The energy and momentum density components for Møller energy-momentum complex are obtained as

$$
\begin{aligned}
& T_{0}^{0}=T_{2}^{0}=T_{3}^{0}=0, \\
& T_{1}^{0}=-\frac{(\alpha+\beta)}{4 \pi} \dot{A} B C e^{(\alpha+\beta) x} .
\end{aligned}
$$

\section{Results and Discussion}

One can note from the results of the energy and momentum densities for the DB Universes in different well known prescriptions as obtained in the previous section that the energy density in Møller prescription is zero. Also, the momentum density components $T_{2}^{0}$ and $T_{3}^{0}$ in Møller prescription vanish identically. However, one of the momentum components in this prescription comes out to be nonvanishing and depends on the sum of the exponents $\alpha$ and $\beta$. In all other cases, the energy and momentum do not vanish identically and depend on $\alpha+\beta$. It is interesting to note that the momentum densities for the DB Universes are the same for the Einstein and Bergmann-Thompson prescriptions. Also, the magnitude of the energy density in these two prescriptions is the same. The energy density for the said model is negative in the Landau-Lifshitz, Papapetrou, and the Bergmann-Thompson prescriptions for nonzero values of the sum $\alpha+\beta$.

In a recent work, Tripathy et al. [57] have calculated the energy and momentum of $\mathrm{BVI}_{h}$ Universes in Einstein, Landau-Lifshitz, Papapetrou, and Bergmann-Thompson prescriptions. In that work, the authors have observed that the energy and momentum of the Universe vanish for a specific choice, $h=-1$, of the exponent $h$. This result raised an obvious question: why is $h=-1$ in $\mathrm{BVI}_{h}$ Universes so special? In order to derive a conclusive remark on this result, we have calculated the energy and momentum of some diagonal Bianchi type Universes described by Bianchi type I (BI), Bianchi type III (BIII), Bianchi type V (BV), and Bianchi type $\mathrm{VI}_{h}\left(\mathrm{BVI}_{h}\right)$ metrics. These metrics can be obtained from the general DB Universe as in (1) with suitable choices of the exponents $\alpha$ and $\beta$ :

(1) For $\alpha=\beta=0$, we get BI metric:

$$
d s^{2}=-d t^{2}+A^{2}(t) d x^{2}+B^{2}(t) d y^{2}+C^{2}(t) d z^{2} .
$$

(2) For $\alpha=h$ and $\beta=0$, we obtain BIII metric:

$$
d s^{2}=-d t^{2}+A^{2}(t) d x^{2}+B^{2}(t) e^{2 h x} d y^{2}+C^{2}(t) d z^{2} .
$$

(3) BV metric can be obtained for $\alpha=\beta=h$ :

$$
\begin{aligned}
d s^{2}= & -d t^{2}+A^{2}(t) d x^{2} \\
& +e^{2 h x}\left[B^{2}(t) d y^{2}+C^{2}(t) d z^{2}\right] .
\end{aligned}
$$

(4) For $\alpha=1$ and $\beta=h$, we get $\mathrm{BVI}_{h}$ metric:

$$
\begin{aligned}
d s^{2}= & -d t^{2}+A^{2}(t) d x^{2}+B^{2}(t) e^{2 x} d y^{2} \\
& +C^{2}(t) e^{2 h x} d z^{2} .
\end{aligned}
$$

In the above equations, the exponent $h$ is considered to assume any real value compatible with the physical Universe. The energy and momentum densities of these DB Universes are given in Tables 3 and 4. The energy and momentum of BI Universe have been obtained earlier by Aydogdu and Salti [46], Banerjee and Sen [58], and Xulu [59]. Recently, Tripathy et al. have obtained the energy and momentum for $\mathrm{BVI}_{h}$ Universes [57]. Our results are in agreement with the results of these earlier works. One can note that the energy and momentum of BI Universe vanish identically for all prescriptions considered in this work. In case of $\mathrm{BVI}_{h}$ Universes, the energy and momentum vanish when $h=-1$. Similarly, for BIII and BV Universes, the energy and momentum vanish for $h=0$. In other words, except for BI Universe, all the prescriptions provide similar results for certain specific value of the metric parameter $h$. The specific choice of the parameter $h$ is different for different DB Universe.

In his interesting work, Tryon [52] assumed that the Universe has appeared from nowhere about $10^{10}$ years ago. As per his thought, at the time of creation of the Universe, the conventional laws of physics may not have been violated. $\mathrm{He}$ proposed a Big Bang model in which the Universe emerged from a large-scale quantum fluctuation of the vacuum. His model predicted a Universe that is homogeneous, isotropic, and closed consisting of equal amount of matter and antimatter. It is worth mentioning here that the Big Bang model and the consequent predictions are consistent with the observations from cosmic microwave background (CMB) radiation. In order to emphasize his thought, Tryon proposed a remarkable conjecture on the energy-momentum of the Universe according to which the Universe must have a zero net value for all conserved quantities. In the same paper, he has mentioned that any closed Universe should have zero net energy. He substantiated his idea by arguments. Many authors have claimed that the laws of physics could have created the Universe from nothing [53, 72-74]. Xulu [75] studied energy and momentum in BI Universe and his results supported the conjecture of Tryon. Berman has also shown that the Robertson-Walker Universe and any other Machian ones have zero total energy [76-78].

More or less, it is now an accepted fact that our Universe is created out of nothing and its net energy is zero. If this 
TABLE 3: Energy and momentum densities for $\mathrm{BI}$ and $\mathrm{BVI}_{h}$ Universes.

\begin{tabular}{|c|c|c|}
\hline \multirow{2}{*}{ Prescription } & \multicolumn{2}{|c|}{ Energy and momentum densities } \\
\hline & BI Universe & $\mathrm{BVI}_{h}$ Universe \\
\hline Einstein & $\Theta_{0}^{0}=\Theta_{1}^{0}=\Theta_{2}^{0}=\Theta_{3}^{0}=0$ & $\begin{array}{l}\Theta_{0}^{0}=\frac{(1+h)^{2}}{8 \pi} \frac{B C}{A} e^{(1+h) x}, \\
\Theta_{1}^{0}=\frac{1+h}{8 \pi} A B C\left(\frac{\dot{B}}{B}+\frac{\dot{C}}{C}\right) e^{(1+h) x}, \\
\Theta_{2}^{0}=\Theta_{3}^{0}=0 .\end{array}$ \\
\hline Landau-Lifshitz & $L^{00}=L^{10}=L^{20}=L^{30}=0$ & $\begin{aligned} L^{00} & =-\frac{(1+h)^{2}}{4 \pi} B^{2} C^{2} e^{2(1+h) x}, \\
L^{10} & =\frac{1+h}{4 \pi} B C(\dot{B} C+B \dot{C}) e^{2(1+h) x}, \\
L^{20} & =L^{30}=0\end{aligned}$ \\
\hline Papapetrou & $\Sigma^{00}=\Sigma^{10}=\Sigma^{20}=\Sigma^{30}=0$ & $\begin{aligned} \Sigma^{00}= & -\frac{(1+h)^{2}}{16 \pi}\left(1+\frac{1}{A^{2}}\right) A B C e^{(1+h) x}, \\
\Sigma^{10}= & \frac{1+h}{16 \pi}\left(\frac{\dot{A}}{A}+\frac{\dot{B}}{B}+\frac{\dot{C}}{C}\right) A B C e^{(1+h) x} \\
& +\frac{1+h}{16 \pi}\left(\frac{1}{A^{2}}\right)\left(\frac{\dot{B}}{B}+\frac{\dot{C}}{C}-\frac{\dot{A}}{A}\right) A B C e^{(1+h) x}, \\
\Sigma^{20}= & \Sigma^{30}=0 .\end{aligned}$ \\
\hline Bergmann-Thompson & $\mathbf{B}^{00}=\mathbf{B}^{10}=\mathbf{B}^{20}=\mathbf{B}^{30}=0$ & $\begin{array}{l}\mathbf{B}^{00}=-\frac{(1+h)^{2}}{8 \pi} \frac{B C}{A} e^{(1+h) x} \\
\mathbf{B}^{10}=\frac{1+h}{8 \pi} \frac{B C}{A}\left(\frac{\dot{B}}{B}+\frac{\dot{C}}{C}\right) e^{(1+h) x} \\
\mathbf{B}^{20}=\mathbf{B}^{30}=0\end{array}$ \\
\hline Møller & $T_{0}^{0}=T_{1}^{0}=T_{2}^{0}=T_{3}^{0}=0$ & $\begin{array}{l}T_{0}^{0}=T_{2}^{0}=T_{3}^{0}=0, \\
T_{1}^{0}=-\frac{1+h}{4 \pi} \dot{A} B C e^{(1+h) x}\end{array}$ \\
\hline
\end{tabular}

TABLE 4: Energy and momentum densities for BIII and BV Universes.

\begin{tabular}{|c|c|c|}
\hline \multirow{2}{*}{ Prescription } & \multicolumn{2}{|c|}{ Energy and momentum densities } \\
\hline & BIII Universe & BV Universe \\
\hline Einstein & $\begin{array}{l}\Theta_{0}^{0}=\frac{h^{2}}{8 \pi} \frac{B C}{A} e^{h x}, \\
\Theta_{1}^{0}=\frac{h}{8 \pi} A B C\left(\frac{\dot{B}}{B}+\frac{\dot{C}}{C}\right) e^{h x}, \\
\Theta_{2}^{0}=\Theta_{3}^{0}=0 .\end{array}$ & $\begin{array}{l}\Theta_{0}^{0}=\frac{h^{2}}{2 \pi} \frac{B C}{A} e^{2 h x}, \\
\Theta_{1}^{0}=\frac{h}{4 \pi} A B C\left(\frac{\dot{B}}{B}+\frac{\dot{C}}{C}\right) e^{2 h x}, \\
\Theta_{2}^{0}=\Theta_{3}^{0}=0 .\end{array}$ \\
\hline Landau-Lifshitz & $\begin{aligned} L^{00} & =-\frac{h^{2}}{4 \pi} B^{2} C^{2} e^{2 h x}, \\
L^{10} & =\frac{h}{4 \pi} B C(\dot{B} C+B \dot{C}) e^{2 h x}, \\
L^{20} & =L^{30}=0 .\end{aligned}$ & $\begin{aligned} L^{00} & =-\frac{h^{2}}{\pi} B^{2} C^{2} e^{4 h x}, \\
L^{10} & =\frac{h}{2 \pi} B C(\dot{B} C+B \dot{C}) e^{4 h x}, \\
L^{20} & =L^{30}=0 .\end{aligned}$ \\
\hline Papapetrou & $\begin{aligned} \Sigma^{00}= & -\frac{h^{2}}{16 \pi}\left(1+\frac{1}{A^{2}}\right) A B C e^{h x}, \\
\Sigma^{10}= & \frac{h}{16 \pi}\left(\frac{\dot{A}}{A}+\frac{\dot{B}}{B}+\frac{\dot{C}}{C}\right) A B C e^{h x} \\
& +\frac{h}{16 \pi}\left(\frac{1}{A^{2}}\right)\left(\frac{\dot{B}}{B}+\frac{\dot{C}}{C}-\frac{\dot{A}}{A}\right. \\
\Sigma^{20}= & \Sigma^{30}=0 .\end{aligned}$ & $\begin{aligned} \Sigma^{00}= & -\frac{h^{2}}{4 \pi}\left(1+\frac{1}{A^{2}}\right) A B C e^{2 h x}, \\
\Sigma^{10}= & \frac{h}{8 \pi}\left(\frac{\dot{A}}{A}+\frac{\dot{B}}{B}+\frac{\dot{C}}{C}\right) A B C e^{2 h x} \\
& +\frac{h}{8 \pi}\left(\frac{1}{A^{2}}\right)\left(\frac{\dot{B}}{B}+\frac{\dot{C}}{C}-\frac{\dot{A}}{A}\right) A B C e^{2 h x}, \\
\Sigma^{20}= & \Sigma^{30}=0 .\end{aligned}$ \\
\hline Bergmann-Thompson & $\begin{aligned} \mathbf{B}^{00} & =-\frac{h^{2}}{8 \pi} \frac{B C}{A} e^{h x}, \\
\mathbf{B}^{10} & =\frac{h}{8 \pi} \frac{B C}{A}\left(\frac{\dot{B}}{B}+\frac{\dot{C}}{C}\right) e^{h x}, \\
\mathbf{B}^{20} & =\mathbf{B}^{30}=0 .\end{aligned}$ & $\begin{array}{l}\mathbf{B}^{00}=-\frac{h^{2}}{2 \pi} \frac{B C}{A} e^{2 h x}, \\
\mathbf{B}^{10}=\frac{h}{4 \pi} \frac{B C}{A}\left(\frac{\dot{B}}{B}+\frac{\dot{C}}{C}\right) e^{2 h x}, \\
\mathbf{B}^{20}=\mathbf{B}^{30}=0 .\end{array}$ \\
\hline Møller & $\begin{array}{l}T_{0}^{0}=T_{2}^{0}=T_{3}^{0}=0 \\
T_{1}^{0}=-\frac{h}{4 \pi} \dot{A} B C e^{h x}\end{array}$ & $\begin{array}{l}T_{0}^{0}=T_{2}^{0}=T_{3}^{0}=0 \\
T_{1}^{0}=-\frac{h}{2 \pi} \dot{A} B C e^{2 h x}\end{array}$ \\
\hline
\end{tabular}


conjecture is to be valid, then all prescriptions for energymomentum should agree with null values of the energy and momentum densities. Keeping this in view, it can be inferred that a flat model is necessary for any diagonal Bianchi type Universe. We have substantiated this fact in our calculation. For DB Universes represented by metric (1), vanishing of the total energy of the Universe requires that the sum $\alpha+\beta$ should vanish; that is, $\alpha+\beta=0$. According to this simple rule, the energy and momentum of BI Universe are zero as this Universe is described by a metric where $\alpha=\beta=0$. Similarly, BIII and BV Universes require the exponent $h$ to vanish. In this context, the question raised in the work of Tripathy et al. [57], that is, "why is $h=-1$ in Bianchi $\mathrm{VI}_{h}$ Universes so special?," can now be answered. In order to satisfy Tryon's conjecture, in $\mathrm{BVI}_{h}$ Universe for which $\alpha=1$ and $\beta=h$, it is necessary that $h$ should be -1 .

\section{Summary}

In the present work, we have obtained the energy and momentum for diagonal Bianchi type Universes in some well known prescriptions such as Einstein, Landau-Lifshitz, Papapetrou, Bergmann-Thompson, and Møller energymomentum complexes in GR. It is observed that Møller energy vanishes for such Universes. However, in all other cases, the energy and momentum of the Universe depend upon the sum $\alpha+\beta$ of the metric parameters $\alpha$ and $\beta$. Momentum densities in the Einstein and the BergmannThompson energy-momentum complexes are the same. Also, the magnitude of energy density is the same for these two prescriptions. The general results of the DB Universes have been used to calculate the energy and momentum of BI, BIII, BV, and $\mathrm{BVI}_{h}$ Universes. All the prescriptions for energy-momentum used in this work provide identical results for the BI Universe. However, for BIII and BV Universes, reasonable results can be obtained when $h=0$. Similarly, for $\mathrm{BVI}_{h}$ Universes, we require $h=-1$ to draw similar conclusion. With these specific choices of the metric parameter $h$, the net energy of the Universes becomes zero.

In a recent work, Tripathy et al. [57] have calculated the energy and momentum of $\mathrm{BVI}_{h}$ Universes and based upon their results they have raised a question: "why is the case $h=$ -1 so special?" In the present work, we have investigated that question keeping in view Tryon's conjecture that advocates a zero total energy of the Universe. In order to obey the conjecture, the diagonal Bianchi type Universes described through the metric in (1) should have a vanishing sum of the exponents $\alpha$ and $\beta$; that is, $\alpha+\beta=0$. Therefore, the energy and momentum for BI Universe identically vanish in all the prescriptions. For BIII and BV Universes, the exponent $h$ requires a value of 0 whereas for $\mathrm{BVI}_{h}$ Universes the exponent $h$ requires a value of -1 . Even if the zero total energy of the Universe or the creation of the present Universe from nothing has been an accepted fact in the context of Big Bang cosmology, still it remains as open problem and a subject of intense debate. Many other cosmological models other than Big Bang models have been proposed in recent times. In view of this, our results are important in the sense that they may provide some insights into the old and unsettled problem of energy-momentum calculation. It is certain that, under the purview of General Relativity, all the prescriptions should provide the same result for the energy of the Universe and, in this sense, anisotropic Bianchi type Universes must also be consistent with that.

\section{Disclosure}

A part of this collaborative research work was done during a workshop conducted by IGIT Physics Forum on General Relativity, Gravitation and Cosmology held at Indira Gandhi Institute of Technology, Sarang, Odisha (India).

\section{Competing Interests}

The authors declare that there are no competing interests regarding the publication of this paper.

\section{References}

[1] A. Einstein, "Zur allgemeinen Relativitatstheorie," Sitzungsberichte der Preussischen Akademie der Wissenschaften, Berlin (Mathematical Physics), vol. 44, no. 2, pp. 778-786, 1915.

[2] R. C. Tolman, Relativity, Thermodynamics and Cosmology, Oxford University Press, Oxford, UK, 1934.

[3] A. Papapetrou, "Einstein's theory of gravitation and flat space," Proceedings of the Royal Irish Academy, vol. 52, p. 11, 1948.

[4] L. D. Landau and E. M. Lifshitz, The Classical Theory of Fields, Addison-Wesley Press, Reading, Mass, USA, 1951.

[5] P. G. Bergmann and R. Thomson, "Spin and angular momentum in general relativity," Physical Review, vol. 89, no. 2, pp. 400-407, 1953.

[6] S. Weinberg, Gravitation and Cosmology: Principles and Applications of General Theory of Relativity, John Wiley \& Sons, New York, NY, USA, 1972.

[7] C. Møller, "On the localization of the energy of a physical system in the general theory of relativity," Annals of Physics, vol. 4, no. 4, pp. 347-371, 1958.

[8] A. Komar, "Covariant conservation laws in general relativity," Physical Review, vol. 113, no. 3, pp. 934-936, 1959.

[9] R. Penrose, "Quasi-local mass and angular momentum in general relativity," Proceedings of the Royal Society of London A: Mathematical and Physical Sciences, vol. 381, no. 1780, pp. 53-63, 1982.

[10] C. W. Misner, K. S. Thorne, and J. A. Wheeler, Graviation, W. H. Freeman and Company, New York, NY, USA, 1973.

[11] F. I. Cooperstock and R. S. Sarracino, "The localisation of energy in general relativity," Journal of Physics A: Mathematical and General, vol. 11, no. 5, pp. 877-883, 1978.

[12] H. Bondi, "Conservation and non-conservation in general relativity," Proceedings of the Royal Society A: Mathematical, Physical and Engineering Sciences, vol. 427, no. 1873, pp. 249258, 1990.

[13] K. S. Virbhadra, "Naked singularities and Seifert's conjecture," Physical Review D, vol. 60, no. 10, Article ID 104041, 6 pages, 1999.

[14] N. Rosen and K. S. Virbhadra, "Energy and momentum of cylindrical gravitational waves," General Relativity and Gravitation, vol. 25, no. 4, pp. 429-433, 1993. 
[15] K. S. Virbhadra, "Energy and momentum of cylindrical gravitational waves-II," Pramana, vol. 45, no. 2, pp. 215-219, 1995.

[16] K. S. Virbhadra, "Energy associated with a Kerr-Newman black hole," Physical Review D, vol. 41, no. 4, pp. 1086-1090, 1990.

[17] K. S. Virbhadra, "Angular momentum distribution in KerrNewman spacetime," Physical Review D, vol. 42, article 1066, 1990.

[18] K. S. Virbhadra, "Energy distribution in Kerr-Newman spacetime in Einstein's as well as Møller's prescriptions," Physical Review D, vol. 42, no. 8, pp. 2919-2921, 1990.

[19] K. S. Virbhadra and J. C. Parikh, "Gravitational energy of a stringy charged black hole," Physics Letters B, vol. 317, no. 3, pp. 312-314, 1993.

[20] K. S. Virbhadra and J. C. Parikh, "A conformal scalar dyon black hole solution," Physics Letters B, vol. 331, no. 3-4, pp. 302-304, 1994.

[21] J. M. Aguirregabiria, A. Chamorro, and K. S. Virbhadra, "Energy and angular momentum of charged rotating black holes," General Relativity and Gravitation, vol. 28, no. 11, pp. 1393-1400, 1996.

[22] E. C. Vagenas, "Energy distribution in the dyadosphere of a Reissner-Nördstrom black hole in Møller's prescription," Modern Physics Letters A, vol. 21, no. 25, pp. 1947-1956, 2006.

[23] E. C. Vagenas, "Energy distribution in a BTZ black hole spacetime," International Journal of Modern Physics D, vol. 14, Article ID 03n04, pp. 573-585, 2005.

[24] T. Multamäki, A. Putaja, I. Vilja, and E. C. Vagenas, "Energymomentum complexes in $f(R)$ theories of gravity," Classical and Quantum Gravity, vol. 25, no. 7, Article ID 075017, 11 pages, 2008.

[25] S. S. Xulu, "Tolman energy of a stringy charged black hole," International Journal of Theoretical Physics, vol. 37, no. 6, pp. 1773-1777, 1998.

[26] S. S. Xulu, "Energy distribution of charged dilaton black holes," International Journal of Modern Physics D, vol. 7, no. 5, pp. 773777, 1998.

[27] S. S. Xulu, "Møller energy for the Kerr-Newman metric," Modern Physics Letters A, vol. 15, no. 24, pp. 1511-1517, 2000.

[28] I. Radinschi, "The energy distribution of the Bianchi type VI0 universe," Chinese Journal of Physics, vol. 39, no. 3, p. 231, 2001.

[29] M. Sharif, "Energy and momentum in space time homogeneous Godel type metrics," International Journal of Modern Physics D, vol. 13, pp. 1019-1028, 2004.

[30] V. C. de Andrade, L. C. T. Guillen, and J. G. Pereira, "Gravitational energy-momentum density in teleparallel gravity," Physical Review Letters, vol. 84, no. 20, pp. 4533-4536, 2000.

[31] O. Aydogdu, "Gravitational energy-momentum density in Bianchi type II space-times," International Journal of Modern Physics D, vol. 15, no. 4, pp. 459-468, 2006.

[32] O. Aydogdu and M. Salti, "The momentum 4-vector in bulk viscous Bianchi type-V space-time," Czechoslovak Journal of Physics, vol. 56, no. 8, pp. 789-798, 2006.

[33] A. M. Abbassi and S. Mirshekari, "Energy-momentum density of gravitational waves," International Journal of Modern Physics A, vol. 23, no. 27-28, pp. 4569-4577, 2008.

[34] S. Mirshekari and A. M. Abbassi, "Energy-momentum prescriptions in general spherically symmetric spacetimes," Modern Physics Letters A: Particles and Fields, Gravitation, Cosmology, Nuclear Physics, vol. 24, no. 10, pp. 747-758, 2009.
[35] S. Mirshekari and A. M. Abbassi, "On energy distribution of two space-times with planar and cylindrical symmetries," International Journal of Modern Physics A, vol. 24, no. 4, pp. 789-797, 2009.

[36] P. Halpern and M. Pecorino, "The localized energy distribution of dark energy star solutions," ISRN Astronomy and Astrophysics, vol. 2013, Article ID 939876, 4 pages, 2013.

[37] I.-C. Yang and I. Radinschi, "The energy of the gamma metric in the Møller prescription,” Modern Physics Letters A: Particles and Fields, Gravitation, Cosmology, Nuclear Physics, vol. 17, no. 18, pp. 1159-1163, 2002.

[38] I.-C. Yang and I. Radinschi, "On the difference of energy between the Einstein and Møller prescription," Chinese Journal of Physics, vol. 42, no. 1, pp. 40-44, 2004.

[39] P. K. Sahoo, K. L. Mahanta, D. Goit et al., "Einstein energymomentum complex for a phantom black hole metric," Chinese Physics Letters, vol. 32, no. 2, Article ID 020402, 2015.

[40] R. M. Gad, "Energy distribution of a Gödel-type space-time," Astrophysics and Space Science, vol. 293, no. 4, pp. 453-460, 2004.

[41] R. M. Gad, "Energy distribution of a stringy charged black hole," Astrophysics and Space Science, vol. 295, no. 4, pp. 459-462, 2005.

[42] R. M. Gad, "Energy and momentum associated with a static axially symmetric vacuum spacetime," Modern Physics Letters A: Particles and Fields, Gravitation, Cosmology, Nuclear Physics, vol. 19, no. 24, pp. 1847-1854, 2004.

[43] R. M. Gad, "Energy and momentum associated with solutions exhibiting directional type singularities," General Relativity and Gravitation, vol. 38, no. 3, pp. 417-424, 2006.

[44] T. Vargas, "The energy of the universe in teleparallel gravity," General Relativity and Gravitation, vol. 36, no. 6, pp. 1255-1264, 2004.

[45] M. Salti, "Energy-momentum in the viscous Kasner-type universe in teleparallel gravity," Astrophysics and Space Science, vol. 299, no. 2, pp. 159-166, 2005.

[46] O. Aydogdu and M. Salti, "Energy of the universe in Bianchitype I models in Møller's tetrad theory of gravity," Astrophysics and Space Science, vol. 299, no. 3, pp. 227-232, 2005.

[47] M. Sharif and A. Jawad, "Energy contents of some well-known solutions in teleparallel gravity," Astrophysics and Space Science, vol. 331, no. 1, pp. 257-263, 2011.

[48] R. M. Gad, "Energy and momentum densities of cosmological models, and equation of state $\rho=\mu$, in general relativity and teleparallel gravity," International Journal of Theoretical Physics, vol. 46, no. 12, pp. 3263-3274, 2007.

[49] G. Kiy and S. Aygün, "Higher-dimensional energy-momentum problem for Bianchi types $\mathrm{V}$ and I universes in gravitation theories," International Journal of Geometric Methods in Modern Physics, vol. 12, no. 4, Article ID 1550045, 2015.

[50] S. Aygün, H. Baysal, and I. Tarhan, "Energy momentum of marder universe in teleparallel gravity," International Journal of Theoretical Physics, vol. 46, no. 10, pp. 2607-2616, 2007.

[51] S. Aygün, I. Tarhan, and H. Baysal, "Scalar field theory and energy-momentum problem of Yilmaz-Rosen metric in general relativity and teleparallel gravity," Astrophysics and Space Science, vol. 314, no. 4, pp. 323-330, 2008.

[52] E. P. Tryon, "Is the universe a vacuum fluctuation?" Nature, vol. 246, no. 5433, pp. 396-397, 1973.

[53] M. G. Albrow, "CPT conservation in the oscillating model of the universe,” Nature Physical Science, vol. 241, pp. 56-57, 1973. 
[54] N. Rosen, "The energy of the universe," General Relativity and Gravitation, vol. 26, no. 3, pp. 319-321, 1994.

[55] F. I. Cooperstock and M. Israelit, "The energy of the universe," Foundations of Physics, vol. 25, no. 4, pp. 631-635, 1995.

[56] V. B. Johri, D. Kalligas, G. P. Singh, and C. W. F. Everitt, "Gravitational energy in the expanding universe," General Relativity and Gravitation, vol. 27, no. 3, pp. 313-318, 1995.

[57] S. K. Tripathy, B. Mishra, G. K. Pandey, A. K. Singh, T. Kumar, and S. S. Xulu, "Energy and momentum of Bianchi type VI h universes," Advances in High Energy Physics, vol. 2015, Article ID 705262, 8 pages, 2015.

[58] N. Banerjee and S. Sen, "Einstein pseudotensor and total energy of the universe," Pramana, vol. 49, no. 6, pp. 609-615, 1997.

[59] S. S. Xulu, "Energy distribution in melvin's magnetic universe," International Journal of Modern Physics A, vol. 15, no. 30, pp. 4849-4856, 2000.

[60] O. Aydogdu and M. Salti, "Energy density associated with the Bianchi type-II space-time," Progress of Theoretical Physics, vol. 115, no. 1, pp. 63-71, 2006.

[61] I. Radinschi, "The energy distribution of the Bianchi type I universe," Acta Physica Slovaca, vol. 50, no. 5, pp. 609-612, 2000.

[62] I. Radinschi, "Energy associated with the Bianchi type VI0 Universe," Chinese Journal of Physics, vol. 39, p. 393, 2001.

[63] S. Aygün and I. Tarhan, "Energy-momentum localization for Bianchi type-IV Universe in general relativity and teleparallel gravity," Pramana, vol. 78, no. 4, pp. 531-548, 2012.

[64] G. Hinshaw, J. L. Weiland, R. S. Hill et al., "Five-year Wilkinson Microwave Anisotropy Probe observations: data processing, sky maps, and basic results," The Astrophysical Journal Supplement Series, vol. 180, no. 2, pp. 225-245, 2009.

[65] R. V. Buiny, A. Berera, and T. W. Kephart, "Asymmetric inflation: exact solutions," Physical Review D, vol. 73, Article ID $063529,2006$.

[66] M. Watanabe, S. Kanno, and J. Soda, "Inflationary universe with anisotropic hair," Physical Review Letters, vol. 102, no. 19, Article ID 191302, 2009.

[67] S. K. Tripathy, "Late time acceleration and role of skewness in anisotropic models," Astrophysics and Space Science, vol. 350, no. 1, pp. 367-374, 2014.

[68] P. A. R. Ade, N. Aghanim, C. Armitage-Caplan et al., "Planck 2013 results. I. Overview of products and scientific results," Astronomy \& Astrophysics, vol. 571, article A1, 2014.

[69] P. A. R. Ade, N. Aghanim, C. Armitage-Caplan et al., "Planck 2013 results. XVI. Cosmological parameters," Astronomy \& Astrophysics, vol. 571, article A16, 2014.

[70] P. A. R. Ade, N. Aghanim, C. Armitage-Caplan et al., "Planck 2013 results. XXII. Constraints on inflation," Astronomy \& Astrophysics, vol. 571, article A22, 2014.

[71] P. A. R. Ade, N. Aghanim, C. Armitage-Caplan et al., "Planck 2013 results. XXIV. Constraints on primordial non-Gaussianity," Astronomy \& Astrophysics, vol. 571, article A24, 2014.

[72] L. Krauss, A Universe from Nothing, New York Free Press, New York, NY, USA, 2012.

[73] S. Hawking, A Brief History of Time, Bentham Paperback, New York, NY, USA, 1996.

[74] J. Hebert, “A universe from nothing?" Acts \& Facts, vol. 41, no. 7, pp. 11-13, 2012.

[75] S. S. Xulu, "Total energy of the Bianchi type I universes," International Journal of Theoretical Physics, vol. 39, no. 4, pp. 1153-1161, 2000.
[76] M. S. Berman, Introduction to General Relativity and the Cosmological Constant, Nova Science, New York, NY, USA, 2007.

[77] M. S. Berman, "Is the Universe a white-hole?" Astrophysics and Space Science, vol. 311, no. 4, pp. 359-361, 2007.

[78] M. S. Berman, “On the zero-energy universe," International Journal of Theoretical Physics, vol. 48, no. 11, pp. 3278-3286, 2009. 

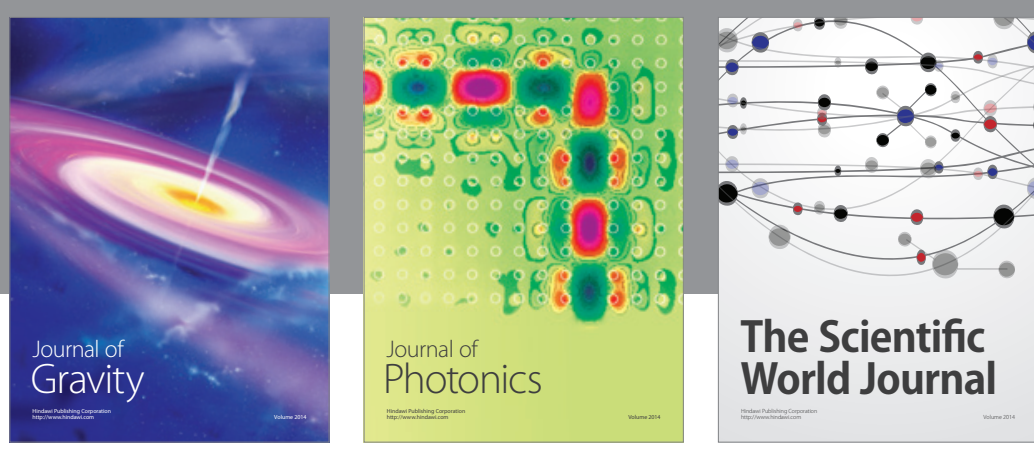

The Scientific World Journal
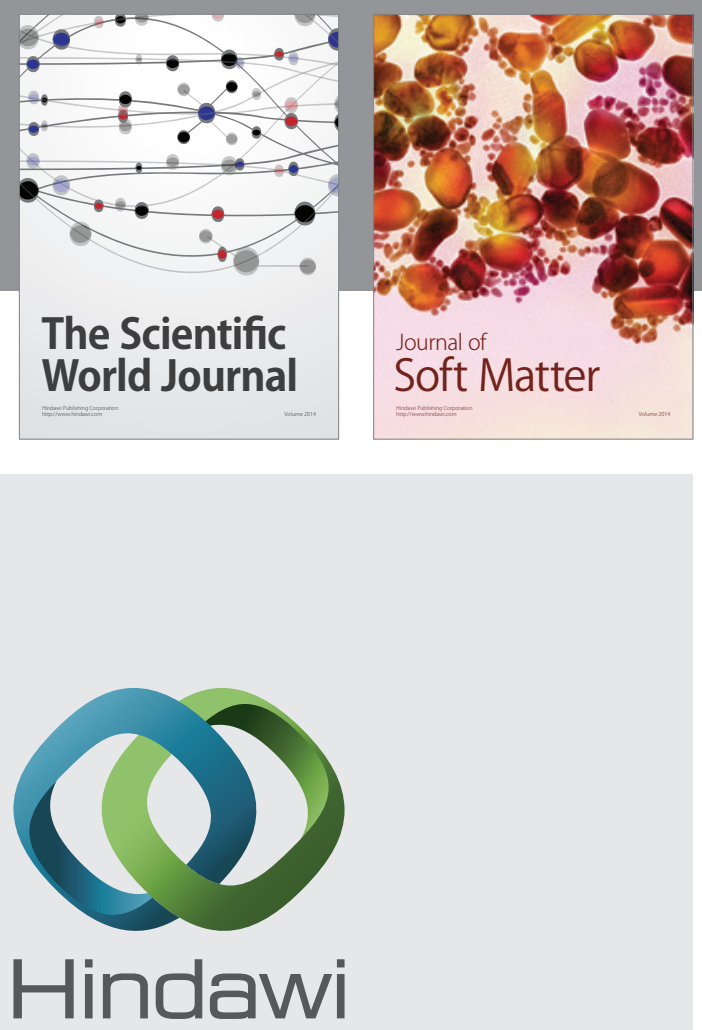

Submit your manuscripts at

http://www.hindawi.com

nternational Journal of

Statistical Mechanics
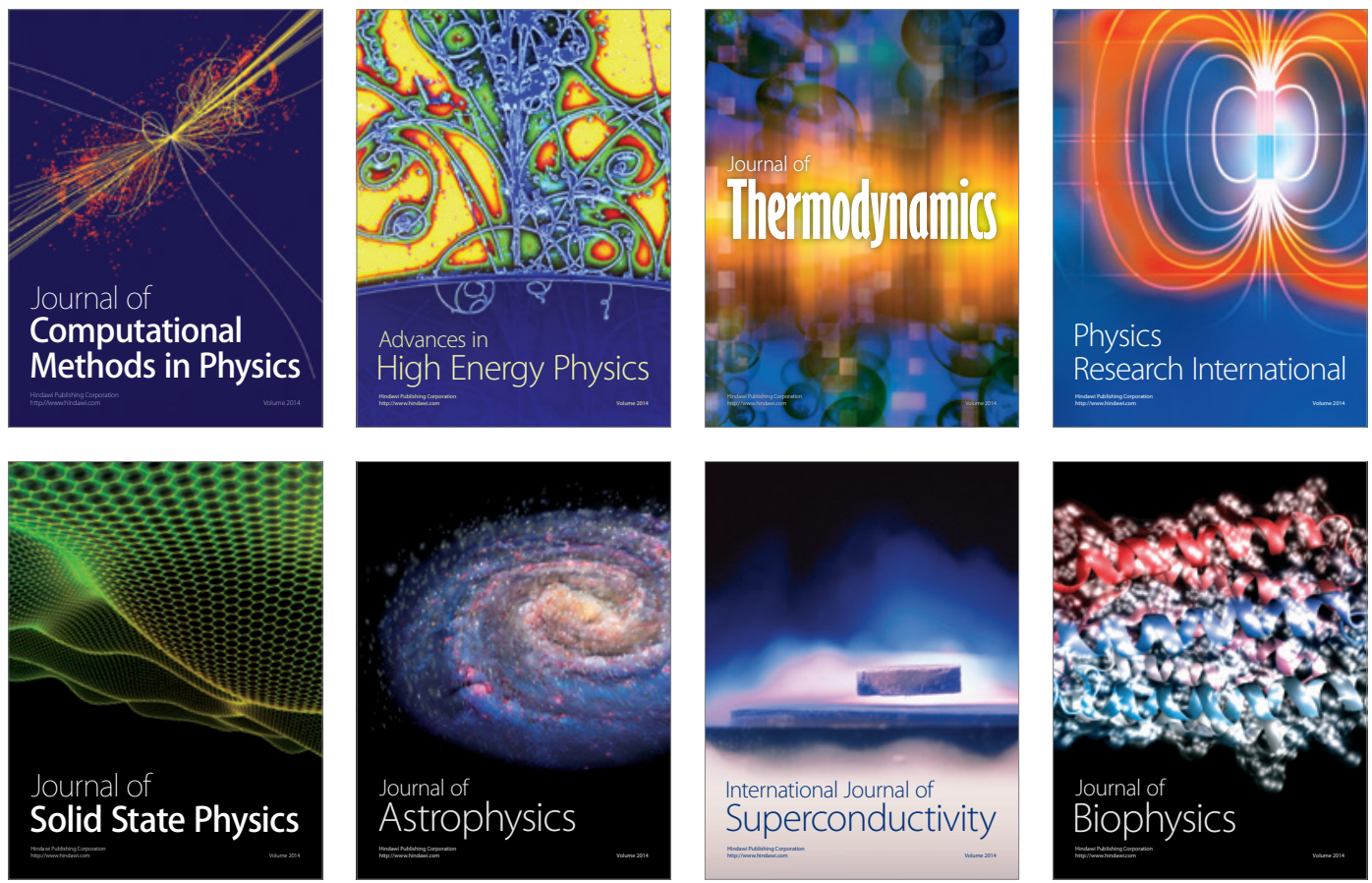
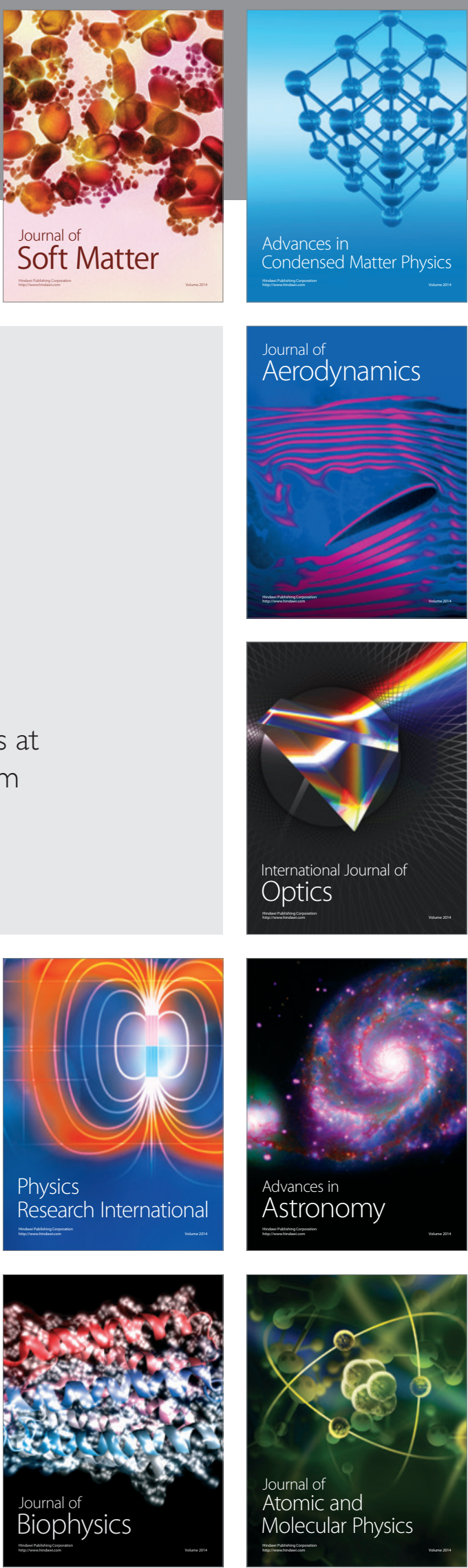